Highly Energetic Physical Processes and

Mechanisms for Emission from Astrophysical Plasmas

IAU Symposium, Vol. 195, 2000

P. C. H. Martens, S. Tsuruta, and M. A. Weber, eds.

\title{
Wave-powered Relativistic Electron Generation in Nonuniform Magnetic Fields
}

\author{
M. P. Leubner \\ Institute for Theoretical Physics, University of Innsbruck, A-6020 \\ Innsbruck, Austria
}

\begin{abstract}
The generation of superthermal electron populations due to resonant energy transfer from Alfvén wave packets can be studied sufficiently well within the Fokker-Planck diffusive formalism, where the particle energization is induced through Landau interaction. From the calculation of the magnetic-field-dependent diffusion operator of an Alfvén wave spectrum, it is demonstrated how resonant energization can be forced in nonuniform magnetic field configurations. On this basis, a mechanism for the acceleration of electrons from thermal to relativistic energies is presented where the specific magnetic field geometry acts as catalyst, providing a highly effective source for synergetic electron energization.
\end{abstract}

Observations indicate that we have to consider particle acceleration as a universal phenomenon, occurring in a large variety of astrophysical plasmas. Those include planetary magnetospheres, the interplanetary medium, solar flares, supernova remnants, and jets in active galaxies. Favored acceleration mechanisms are based on resonant wave-particle interaction, shock heating, and electric fields, where the energy ranges from suprathermal tails in the distribution function up to relativistic energies. To account for energization in the entire energy range, two-stage acceleration by different mechanisms is often proposed, e.g., pre-acceleration by lower hybrid waves and acceleration by cascading fast-mode waves (Miller, LaRosa, \& Moore 1996).

In nonuniform magnetic fields, a unique wave acceleration mechanism may become effective in a broadband spectrum of Alfvén waves where multiple wave packets at different phase velocities provide the energy source. Synergetic effects result in a continuous energization process. Electrons are accelerated resonantly out of the bulk of the distribution such that they become again resonant with a wave packet of higher phase velocity, due to magnetic field inhomogeneities. The analysis can be performed within a diffusive Fokker-Planck approach where the particle energization is induced through Landau interaction. Due to energy transfer in a broadband Alfvén wave spectrum, this method generates a suprathermal electron population in uniform magnetic fields, consistent with the observations of $\kappa$-like distributions in magnetospheric or coronal plasmas (Leubner 2000), where whistler-wave-mode interaction may be important as well (Ma \& Summers 1998); for a Vlasov simulation model, see Viñas, Wong, \& Klimas (2000).

In contrast to such heating mechanisms, the magnetic-field-dependent diffusion coefficient of an Alfvén wave spectrum in a nonuniform magnetic field structure of increasing field strength provides the basis of synergetic electron 
(a)

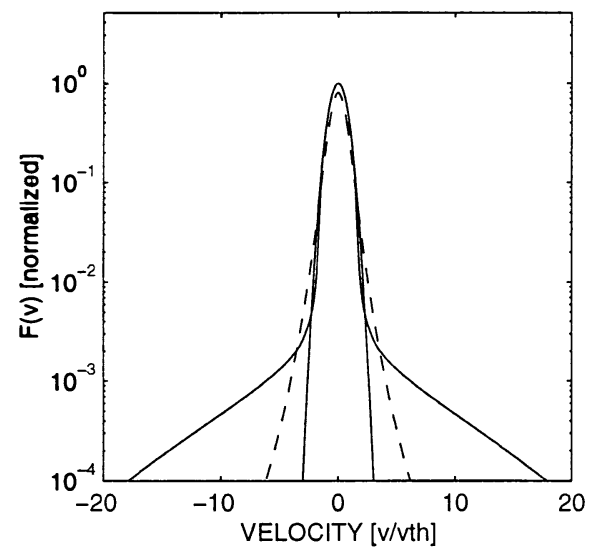

(b)

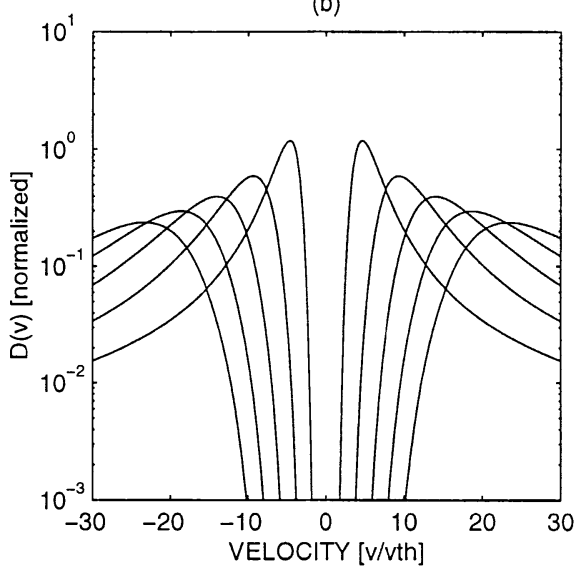

Figure 1. (a) A Maxwellian, a $\kappa$-distribution, and a saturated structure exhibiting highly energetic electron populations. (b) Diffusion operator in increasing magnetic fields from $500 \mathrm{G}$ (innermost curves) to $2500 \mathrm{G}$ (outermost curves). Velocities are normalized to the thermal speed $v_{\text {th }}$.

acceleration, which may operate in various astrophysical acceleration regions. Taking a Maxwellian and solar flare parameters as initial conditions, Figure 1a displays the resulting saturated stage - a highly energetic structure after completion of the time evolution of the velocity space distribution. A Crank-Nicholson implicit discretization is applied for the Fokker-Planck simulation where the diffusion operator is modeled by a broadband wave spectrum and the system of equations is closed by the dispersion relation for kinetic-shear Alfvén waves. Efficient acceleration from coronal up to relativistic energies can be achieved uniquely, without pre-acceleration by other mechanisms, generating a significant percentage of highly energetic electrons. Snapshots of the corresponding evolution of the diffusion operator, due to an increasing magnetic field geometry between 500-2500 G, are displayed in Figure 1b. For comparison with heating mechanisms in uniform magnetic fields, the dashed lines in Figure 1a represent a $\kappa$-distribution-a power law in particle speed with $\kappa=2$.

Summarizing, a nonuniform magnetic field structure acts as catalyst for synergetic effects, generating from a broadband Alfvén wave spectrum, via resonant interaction, a uniform electron acceleration mechanism from thermal up to relativistic energies.

\section{References}

Leubner, M. P. 2000, Planet. Space Sci., submitted

Ma, C., \& Summers, D. 1998, Geophys. Res. Lett., 25, 4099

Miller, J. A., LaRosa, T. N., \& Moore, R. L. 1996, ApJ, 461, 445

Viñas, A. F., Wong, H. K., \& Klimas, A. J. 2000, ApJ, in press 